Kompass

Onkologie

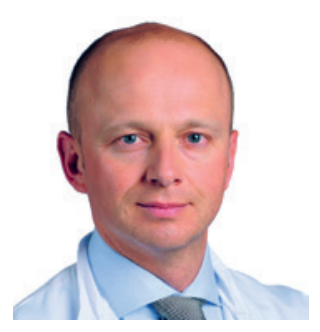

\section{Hubert Kübler}

Direktor der Klinik und Poliklinik für Urologie und Kinderurologie Universitätsklinikum

Würzburg, Würzburg, Deutschland

\title{
Therapie des Hodentumors im 21. Jahrhundert
}

In den letzten Jahren hat das Bewusstsein über die Langzeitfolgen und Spättoxizität der Therapie von Hodentumoren die Therapielandschaft verändert und zu extrem stadienspezifischen Therapiealgorithmen geführt. Durch die im April letzten Jahres publizierte interdisziplinäre S3-Leitlinie zur Diagnostik und Therapie von Hodentumoren ist die Behandlung von Hodentumorpatienten weiter standardisiert worden. Für die frühen Stadien des Hodentumors hat dies zu einer deutlichen Reduktion von adjuvanten Therapiemaßnahmen geführt. So wird beispielsweise für das klassische Seminom im klinischen Stadium I unabhängig von Risikofaktoren die Surveillance empfohlen und die adjuvante Therapie mit Carboplatin nur noch in begründeten Ausnahmefällen in Erwägung gezogen. Entscheidend für die Therapiealgorithmen ist jedoch die korrekte Stadieneinteilung der Patienten, um nicht aus falsch verstandenem Sicherheitsdenken unnötigen Therapieschritte zu initiieren. Wie generell in der Onkologie kommt hier rasch der Ruf nach prädiktiven Markern und molekularen Sig- naturen für die richtige Therapiestratifizierung auf.

MicroRNA sind kurze, nichtcodierende RNAFragmente, die eine wichtige Rolle bei der Genregulation spielen. Dieckmann und Kollegen haben die microRNA-371a-3p als neuen Biomarker in einer prospektiven, multizentrischen Studie an 616 Patienten mit Hodentumoren analysiert und eine beeindruckende Sensitivität und Spezifität $\mathrm{Ihr}$ von über 90\% nachweisen können. Die Markerspiegel korrelierten mit klinischem Stadium, Tumorgröße und Therapieansprechen [1]. Bevor der M371 Test für die klinische Routine zur Verfügung steht, bedürfen Hubert Kübler die Ergebnisse jedoch einer weiteren Validierung.

Wie kein anderes Ereignis nach dem 2. Weltkrieg hat die Corona-Pandemie unser soziokulturelles Leben fundamental mit weitreichenden Konsequenzen auch für die medizinische Versorgung unserer Patienten verändert. Welche weitreichende Bedeutung dies für die Betreuung und Behandlung COVID-19-positiver onkologischer Patienten darstellt, wird exemplarisch an einem
Case Report eines jungen Patienten mit Hodentumor deutlich [2]. Durch die gesteigerte perioperative Morbidität und Mortalität COVID-positiver Patienten sind hier gegebenenfalls alternative Therapieansätze zu verfolgen.

Eine spannende Lektüre der aktuellen Ausgabe von Kompass Onkologie wünscht mit kollegialen Grüßen,

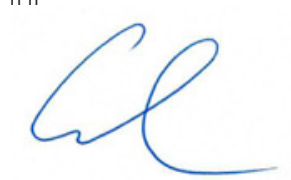

\section{Literatur}

1 Dieckmann KP et al., Serum Levels of MicroRNA-371a-3p (M371 Test) as a New Biomarker of Testicular Germ Cell Tumors: Results of a Prospective Multicentric Study. J Clin Oncol 2019;37:1412-1423

2 Almassi N et al., 'Case of the Month' from Memorial Sloan Kettering Cancer Center, New York, NY, USA: managing newly diagnosed metastatic testicular germ cell tumour in a COVID 19-positive patient. BJU Int 2020; 126:333-335 